\title{
Low-cost photoacoustic imaging systems based on laser diode and light-emitting diode excitation
}

\author{
Qingkai Yao, Yu Ding, Guodong Liu** and Lvming Zeng ${ }^{\dagger}, *$ \\ Key Lab of Optic-Electronic and Communication \\ Jiangxi Sciences and Technology Normal University \\ Nanchang 330038, P. R. China \\ *liuguodong95@163.com \\ †zenglvming@163.com
}

Received 30 March 2017

Accepted 9 May 2017

Published 9 June 2017

\begin{abstract}
Photoacoustic imaging, an emerging biomedical imaging modality, holds great promise for preclinical and clinical researches. It combines the high optical contrast and high ultrasound resolution by converting laser excitation into ultrasonic emission. In order to generate photoacoustic signal efficiently, bulky Q-switched solid-state laser systems are most commonly used as excitation sources and hence limit its commercialization. As an alternative, the miniaturized semiconductor laser system has the advantages of being inexpensive, compact, and robust, which makes a significant effect on production-forming design. It is also desirable to obtain a wavelength in a wide range from visible to nearinfrared spectrum for multispectral applications. Focussing on practical aspect, this paper reviews the state-of-the-art developments of low-cost photoacoustic system with laser diode and light-emitting diode excitation source and highlights a few representative installations in the past decade.
\end{abstract}

Keywords: Photoacoustic imaging; photoacoustic tomography; photoacoustic microscopy; laser diode; light-emitting diode.

\section{Introduction}

In 1880, Alexander Graham Bell ${ }^{1}$ discovered and put forward the photoacoustic effect in solids for the first time. Due to converting absorbed optical energy into a temperature rise, a corresponding pressure change will generate an acoustic signal that can be detected by the ultrasonic receiver, then a highresolution image of optical absorption differences can be reconstructed to reveal the internal structure and function; this is the so-called photoacoustic imaging
(PAI). ${ }^{2-5}$ In general, PAI has three major types: photoacoustic tomography or photoacoustic computed tomography (PAT/PACT), photoacoustic microscopy (PAM), and photoacoustic endoscopy (PAE). ${ }^{6-19}$ In PAT, an expanded pulsed laser beam illuminates the imaging area homogeneously. ${ }^{20-28}$ The laser-induced photoacoustic signal can be acquired using either singleelement scanning or multi-element array detection, which determines the imaging speed and image quality with various time-reversal reconstruction algorithms.

\$Corresponding authors.

This is an Open Access article published by World Scientific Publishing Company. It is distributed under the terms of the Creative Commons Attribution 4.0 (CC-BY) License. Further distribution of this work is permitted, provided the original work is properly cited. 
Unlike PAT, PAM can be divided into acoustic-resolution PAM (AR-PAM) and optical-resolution PAM (OR-PAM), depending on whether the acoustic focus or optical focus is more tightly focussed..$^{29-35}$ The spatial resolution and sensitivity of the imaging systems depend on the laser excitation and acoustic detection configurations. Like PAM, PAE is emerging as a novel technology for imaging internal organs with longer depth. ${ }^{36-41}$ The small photoacoustic endoscopic probe usually includes the function units of laser delivery, acoustic sensing, and mechanical scanning, which could be positioned close enough to the area of interest. Presently, the relatively slow imaging speed is limited by the mechanical rotational scanning for a crosssectional B-scan image.

Normally, the laser pulse duration is needed to be less than both the thermal and stress confinement times, such as nanosecond and femtosecond laser. For decades, PAI has developed a great extent as the development of the laser technology and optimization of the system structure. ${ }^{42-63}$ The PAI systems choose Q-switched Nd:YAG or OPO nanosecond lasers as excitation sources, which are usually expensive and bulky to be used as a handy tool for clinical applications. ${ }^{64}$ As PAI is ceaselessly advancing in the wake of laser technologies, an alternative is to use the rising semiconductor lasers such as laser diodes and light-emitting diodes (LEDs). They have the competitive advantages of being inexpensive, compact, and robust. Furthermore, they commonly have high repetition rate and wide spectrum range for the real-time multispectral applications. ${ }^{65}$ Currently, pulsed laser diodes are commercially available with a hundreds-watt-level power output at near-infrared (NIR) wavelengths, and continuous-wave (CW) LEDs can cover the full visible (VIS) spectrum. ${ }^{66-77}$ They could play a broad role in superficial vascular PAI, especially where the laser energy requirements are relatively modest. Although the relatively weak power represents a main disadvantage for efficient photoacoustic effect, there is still substantial scope to mitigate this for deeper imaging depth by time and frequency methods. In particular, the further promotion of PAE technology will make tremendous impact on the deeper imaging of internal organs. In this review, we focus on the current status and recent progress in PAI systems with the laser diode and LED excitations and highlight studies performed in the past decade.

\section{Laser Diode-Based PAT (LD-PAT)}

\subsection{Pulsed LD-PAT}

Ten years ago, a circular scanning-based LD-PAT system was developed, as shown in Fig. 1(a). ${ }^{78,79} \mathrm{It}$ contained four $905 \mathrm{~nm}$ pulsed laser diodes, which can achieve $175 \mathrm{~W}$ peak output power, $0.1 \%$ duty cycle, and a pulse duration of $500 \mathrm{~ns}$. Each of outputs was coupled to a fiber to irradiate the sample which has the same absorption coefficient with blood. The laser diodes with a pulse duration of $500 \mathrm{~ns}$ were used to excite the photoacoustic signals. A $3.5 \mathrm{MHz}$ focussed transducer revolves around the sample to capture the signals with 100 steps by a stepper motor. The magnification of the amplifier is $40 \mathrm{~dB}$ and the received signal was averaged 5000 times. Figure 1(b) shows the reconstructed image after deconvolving the photoacoustic data with the temporal shape of the pulse duration. The pulsed LD-PAT system achieved a two-dimensional image, which was demonstrated the potential to be used for short-range imaging applications such as visualizing

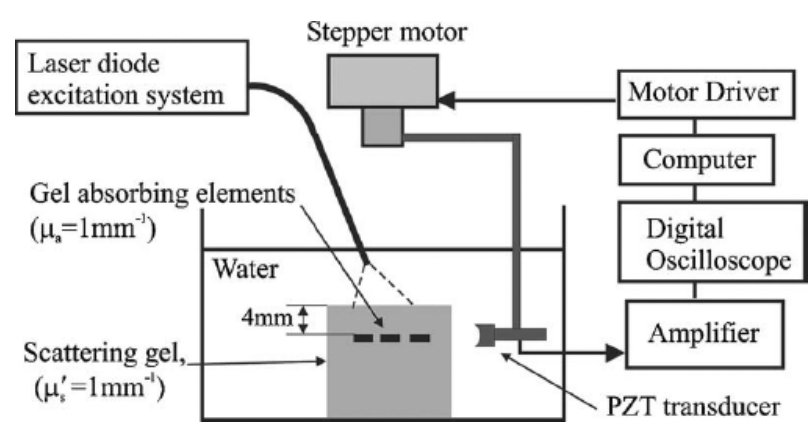

(a)

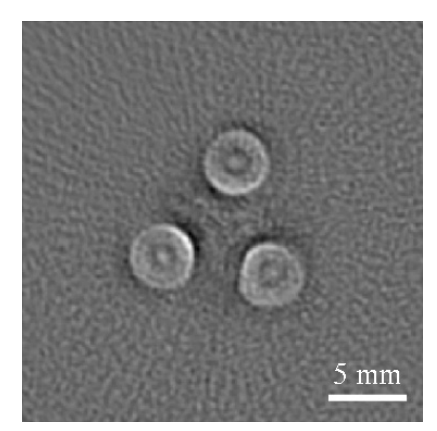

(b)

Fig. 1. (a) Cylindrical scanning LD-PAT system and (b) image obtained with a pulse duration of $500 \mathrm{~ns}$ (images adapted from Refs. 78, 79). 


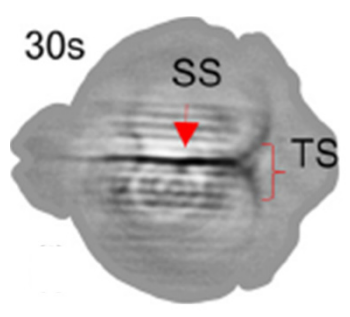

(a)

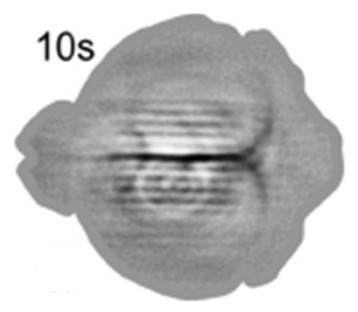

(b)

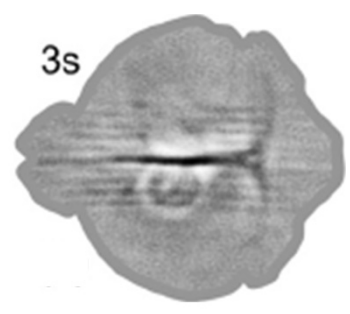

(c)

Fig. 2. In vivo imaging of rat brain in lateral plane at different scanning speeds: (a) $30 \mathrm{~s}$, (b) $10 \mathrm{~s}$, and (c) $3 \mathrm{~s}$, superior sagittal sinus (SS); transverse sinus (TS) (images adapted from Ref. 80).

superficial vascular anatomy. It is considered that the work represents the first attempt in developing a new generation of compact and low-cost excitation system that provides an alternative to solidstate lasers. Before the two mentioned, Kolkman et al. ${ }^{59}$ have shown an in vivo image by coupling the light of the pulsed lasers into a glass fiber. It took initial steps toward LD-based PAI, although the group did not show the configuration of system.

Another similar instrument of pulsed LD-PAT was applied to biomedical imaging in vivo. In this system, an $803 \mathrm{~nm}$ laser diode with pulse energy of $1.3 \mathrm{~mJ}$ and $7 \mathrm{kHz}$ repetition rate was used as the excitation source. ${ }^{80}$ Mice at 6 weeks have been selected for in vivo experiment, and the images of the rat brain were reconstructed by receiving photoacoustic signals in 30,10 , and $3 \mathrm{~s}$ periodic time. The resolution of the pulsed LD-PAT system is up to $380 \mu \mathrm{m}$ with a $2.25 \mathrm{MHz}$ transducer. Figure 2 shows the relationship between the signal-to-noise ratios (SNRs) and scan time. The images of sagittal sinus and transverse sinuses are fairly distinct, and the SNR is low at high-speed imaging because of the low energy pulses. The pulsed LD-PAT system demonstrated that the pulsed laser diode could provide highspeed in vivo imaging for preclinical applications.

\section{2. $C W L D-P A T$}

A $784 \mathrm{~nm}$ intensity-modulated CW laser diode was used for PAT, as shown in Fig. 3(a). ${ }^{43}$ The laser diode was modulated at $2.45 \mathrm{MHz}$ with an output power of $120 \mathrm{~mW}$ and was coupled to a scanning head by an optical fiber. A $2.45 \mathrm{MHz}$ bowl-shaped transducer was used to receive the photoacoustic signals. The reconstructed image of rabbit blood vessel was shown in Fig. 3(b). The photoacoustic signals have an SNR of $43 \mathrm{~dB}$, and the system achieved an axial resolution of $0.45 \mathrm{~mm}$ and a lateral resolution of $1 \mathrm{~mm}$. The CW LD-PAT system can provide the characteristic information of vasculature $\sim 3 \mathrm{~mm}$ deep, but the single-frequency CW measurement misses the time domain information about the flight time of photoacoustic signal.

\subsection{Dual-modality photoacoustic/ ultrasound system}

Figures 4(a) and 4(b) show a clinical imaging system that was composed of ultrasound and PAI modality. ${ }^{81} \mathrm{~A}$ handheld probe is embedded with $805 \mathrm{~nm}$ laser diode arrays, which operated on a pulse energy of $0.56 \mathrm{~mJ}$, repetition frequency of

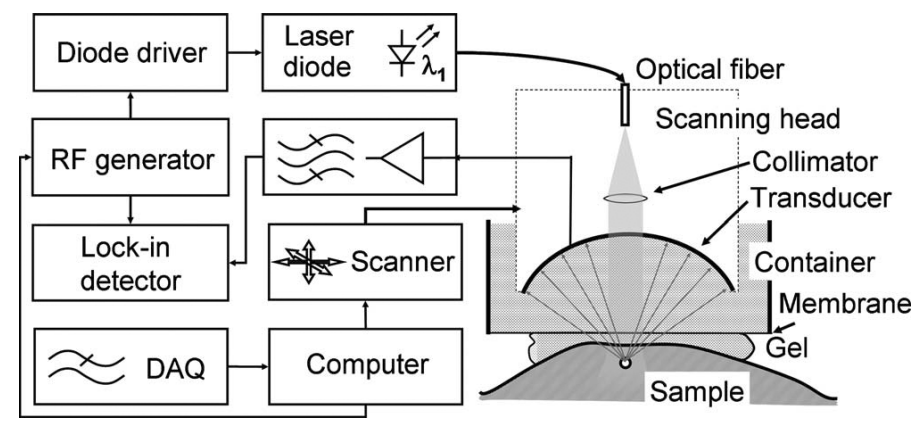

(a)

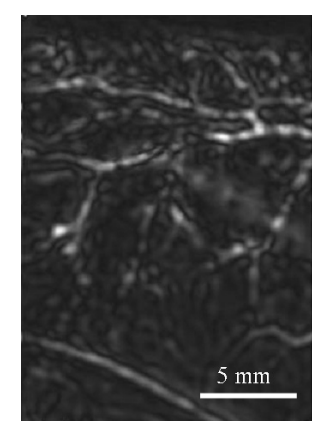

(b)

Fig. 3. (a) Schematic of the CW LD-PAT system and (b) amplitude image of a rabbit ear vasculature (images adapted from Ref. 43). 


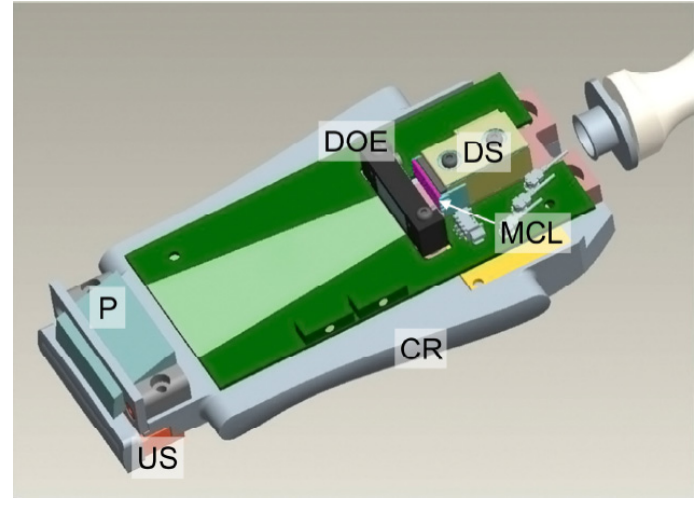

(a)

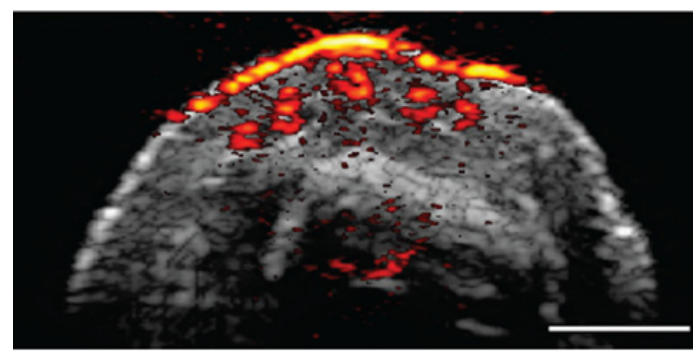

(c)

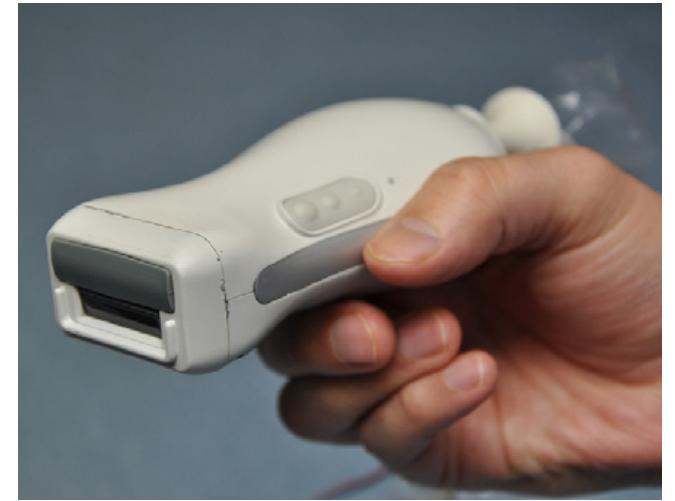

(b)

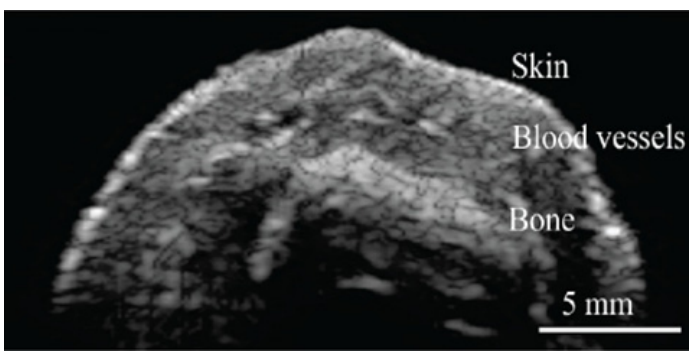

(d)

Fig. 4. (a) Schematic of the handheld probe. US, ultrasound array transducer; P, deflecting prism; DOE, diffractive optical elements; DS, diode stack; MCL, micro-cylindrical lenses; CR, aluminum cooling rim. (b) External shape of the handheld probe, (c) photoacoustic/ultrasound image of a human proximal interphalangeal joint, and (d) ultrasound image of a human proximal interphalangeal joint (images adapted from Ref. 81).

$10 \mathrm{kHz}$, and pulse duration of $130 \mathrm{~ns}$. In addition, the probe combined the reflection mode photoacoustic and the single direction scan. A $7.5 \mathrm{MHz}$ ultrasound array was employed with an acoustic lens to focus in the elevation plane at distance about $20 \mathrm{~mm}$. Figures 4(c) and 4(d) show the ultrasound and photoacoustic images of the human proximal interphalangeal joint, showing a detailed absorption distribution alongside the anatomical structure. The pulsed LD-PAT system achieved an axial resolution of $0.28 \mathrm{~mm}$, lateral resolution about $0.5 \mathrm{~mm}$, and penetration depth up to $15 \mathrm{~mm}$. It can obtain 10 images per second under the maximum imaging speed and each image was averaged 20 frames, which can provide a portable real-time dual-modality imaging for clinical applications. Recently, van den Berg et al. ${ }^{82}$ took further efforts toward commercial probes for preclinical detection of liver fibrosis. Both devices are combined in dual modality $\mathrm{PA} / \mathrm{US}$ imaging, which enables robust noninvasive identification of the liver during experiments and accurate region-of-interest selection.

\section{Laser Diode-Based PAM (LD-PAM)}

\subsection{Pulsed NIR LD-PAM}

A $905 \mathrm{~nm}$ pulsed laser diode operating on the ORPAM model is shown in Fig. 5(a). ${ }^{83,84}$ The laser diode has $140 \mathrm{~W}$ peak power and pulse energy of $4.9 \mu \mathrm{J}$. The optical lens group with a numerical aperture of 0.62 has a focus length of $15 \mathrm{~cm}$. The excitation source was composed of optical lenses and the laser diode, as shown in Fig. 5(b). A $4.53 \mathrm{MHz}$ transducer was used to capture the photoacoustic signals by averaging 128 times. A dead ant with a length of $\sim 3.5 \mathrm{~mm}$ and diameter of $0.7 \mathrm{~mm}$ was selected as the sample. Figures 5(c) and 5(d) show the two-dimensional reconstructed images which displayed characteristics of spatial shape and size. The pulsed NIR LD-PAM system demonstrated that the pulsed NIR laser diode can be employed for biological tissue imaging and may represent a potential development direction for superficial biomedical applications.

Another similar NIR LD-PAM system was used to image ex vivo mouse ear. ${ }^{85}$ In this system, a pulsed 

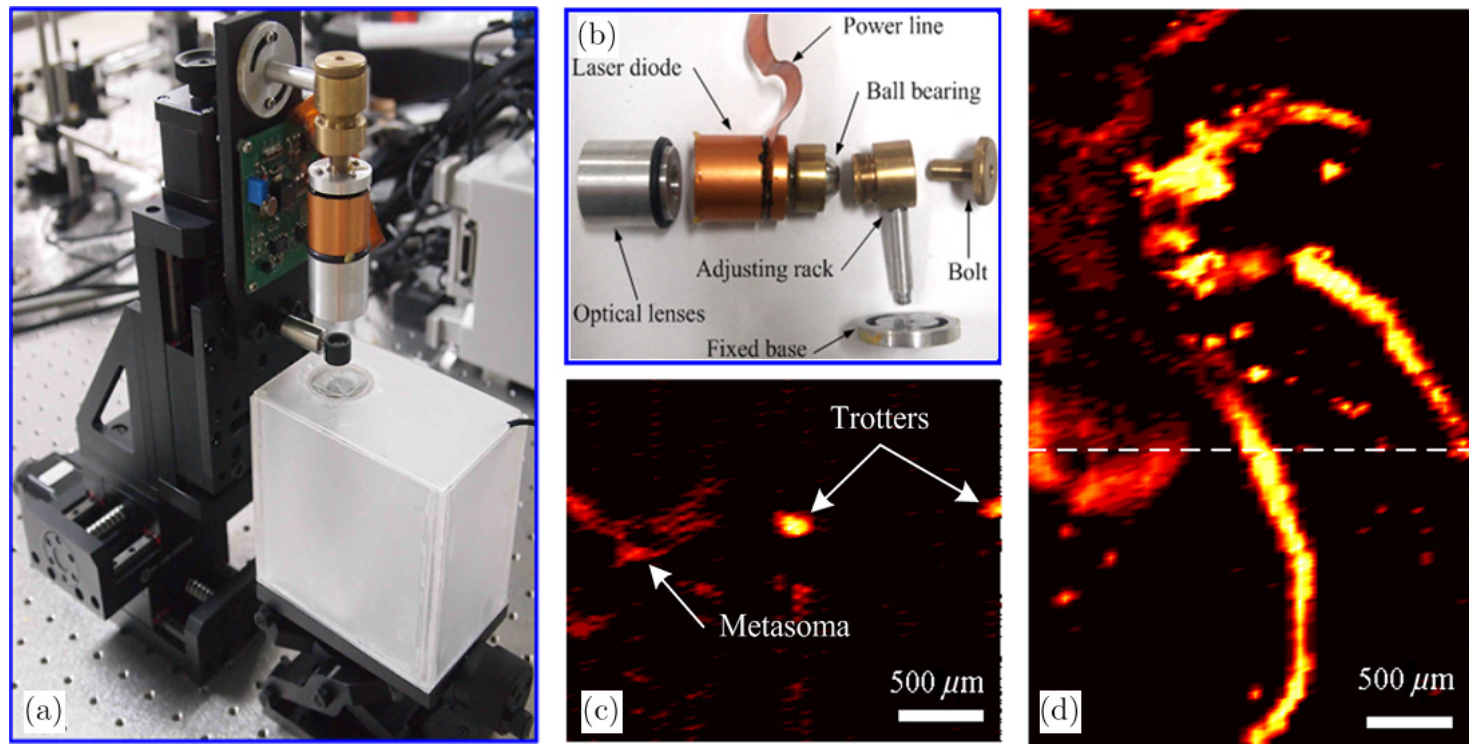

Fig. 5. (a) and (b) Photograph of pulsed NIR LD-PAM system and its laser diode excitation and (c) and (d) photoacoustic images of the ant along the depth and horizontal directions (images adapted from Refs. 83, 84).

laser diode with a wavelength of $905 \mathrm{~nm}$ and $130 \mathrm{~W}$ peak power was used as excitation source. The pulse repetition rate is $1 \mathrm{kHz}$ and the pulse width is $124 \mathrm{~ns}$. Figure 6(a) shows the photoacoustic image of the mouse ear, which contains major blood vessels and network pattern. The photoacoustic signal was performed 128 averaging with a $3.5 \mathrm{MHz}$ transducer. In addition, a $905 \mathrm{~nm} 650 \mathrm{~W}$ pulsed laser diode was exploited for another set of experiment. ${ }^{86}$ The reconstructed image of ex vivo porcine ovary was shown in Fig. 6(b). The imaging depth and SNR are $\sim 1 \mathrm{~mm}$ and $25 \mathrm{~dB}$, respectively. It proved the potential of pulsed NIR LD-PAM system for revealing the characterization of biological tissue and the diagnosis of the tissue cancer.

\subsection{Pulsed VIS LD-PAM}

A $300 \mathrm{~mW}$ VIS laser diode was exploited to the LDPAM system, as shown in Figs. $7(\mathrm{a})-7(\mathrm{c}) .{ }^{87}$ The laser diode has a wavelength of $405 \mathrm{~nm}$ and a pulsed energy of $52 \mathrm{~nJ}$. The pulse duration of the laser

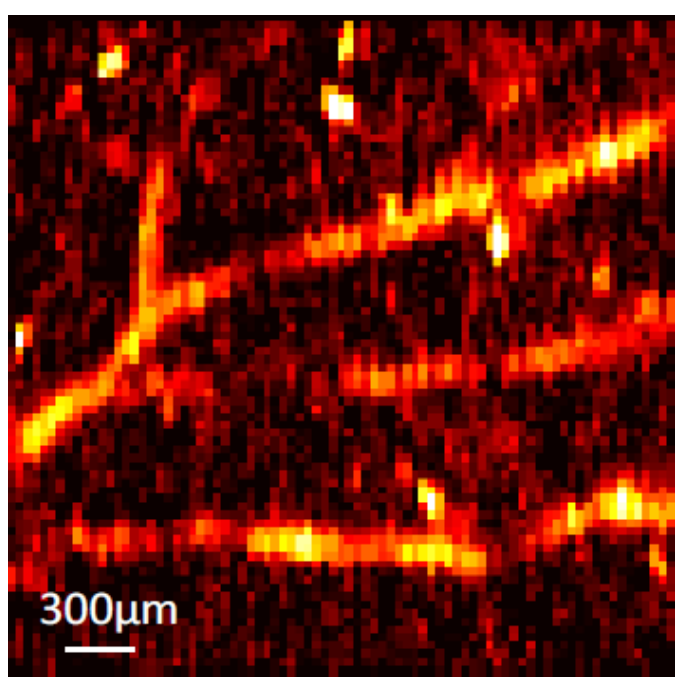

(a)

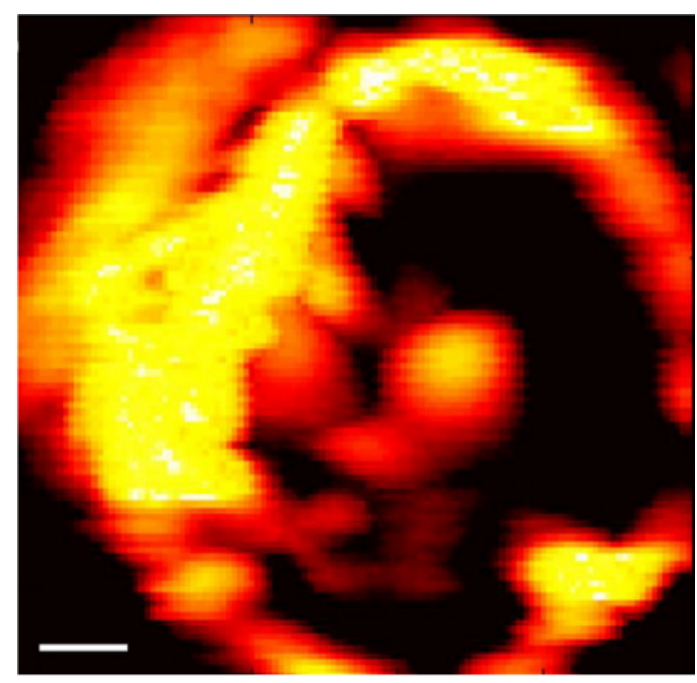

(b)

Fig. 6. (a) and (b) Photoacoustic images of mouse ear and porcine ovary (images adapted from Refs. 85, 86). 



Fig. 7. (a) and (b) Photographs of the compact laser diode and assembled excitation source, (c) schematic of the pulsed VIS LDPAM system, (d) cross-sectional image of the carbon fibers, and (e) ex vivo image of mouse subcutaneous microvasculature (images adapted from Ref. 87).

diode is $174 \mathrm{~ns}$ and the pulse repetition rate can reach $1 \mathrm{kHz}$. A $3.6 \mathrm{MHz}$ transducer was used to obtain the surfaces flaws of carbon fibers in Fig. 7(d), which was acquired by scanning along the $z$-axis with a $5 \mu \mathrm{m}$ step. The SNR of the photoacoustic image can achieve $22 \mathrm{~dB}$ without signal averaging and the lateral resolution reach up to $0.95 \mu \mathrm{m}$. An image of a mouse back ex vivo is shown in Fig. 7(e), which was performed 512 averaging. Without contrast agent, the detailed features of the blood vessels were seen clearly. The pulsed VIS LD-PAM system was verified as a reliable mode for label-free imaging in superficial tissue.

A $405 \mathrm{~nm}$ Blu-ray DVD pickup head was integrated into an OR-PAM system. ${ }^{68}$ The pickup head can generate a tunable pulse duration ranging from 10 to $30 \mathrm{~ns}$ and a pulsed repetition rate of $>30 \mathrm{kHz}$. A $50 \mathrm{MHz}$ transducer was employed to acquire the photoacoustic signals. Figures $8(\mathrm{a})-8(\mathrm{c})$ show the reconstructed images of an unstained human blood smear, the zoom-in of the dashed region, and the maximum amplitude projection, respectively. Under a 1500-time signal averaging, the characteristics of red blood cells were visible clearly with a lateral resolution of $1 \mu \mathrm{m}$, which has a great advantage in subsurface skin chromophores. Overall, the results verified the practicability of the pulsed VIS LD-PAM for label-free imaging in both small animal studies and blood examinations. 


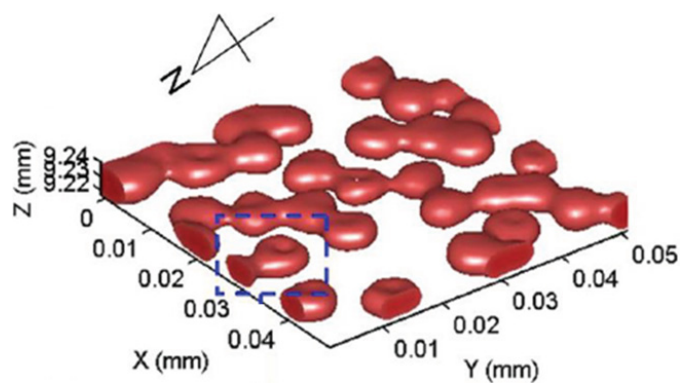

(a)

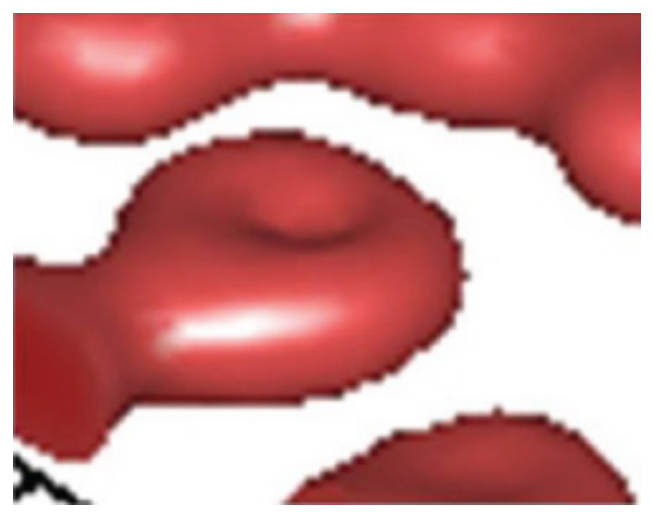

(b)

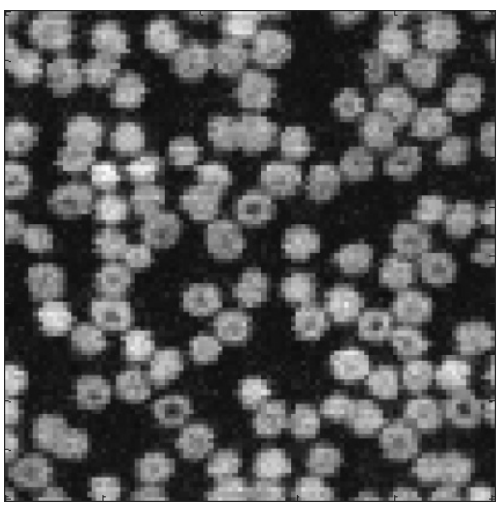

(c)

Fig. 8. (a) Three-dimensional photoacoustic image of an unstained human blood smear, (b) zoom-in of the dashed box in (a), and (c) maximum amplitude projection of the unstained human blood smear (images adapted from Ref. 68).

\section{3. $C W L D-P A M$}

A $405 \mathrm{~nm}$ CW laser diode was utilized to photoacoustic and fluorescence microscopy. ${ }^{88}$ The laser diode was modulated at $6 \mathrm{MHz}$ and another $532 \mathrm{~nm}$

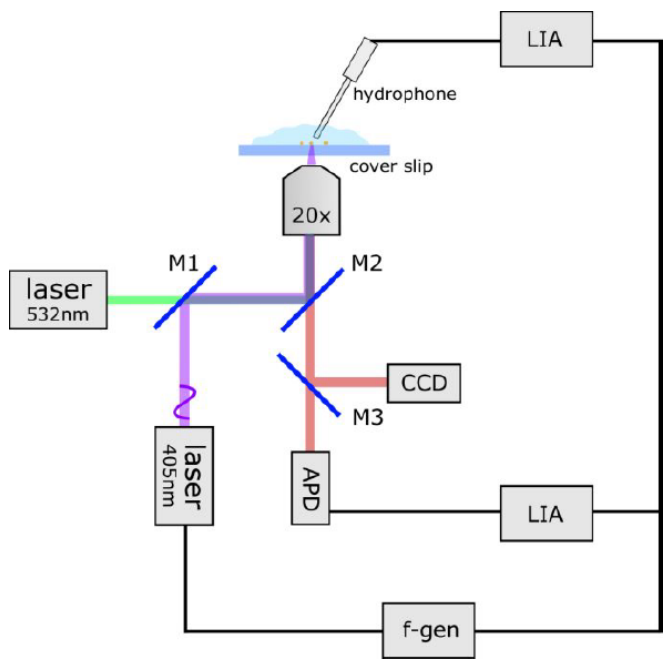

(a)
CW laser diode was used as offset radiation. The schematic of the CW LD-PAM system is shown in Fig. 9(a). The dielectric mirror M2 separates luminescence from the excitation light, and the red reflector

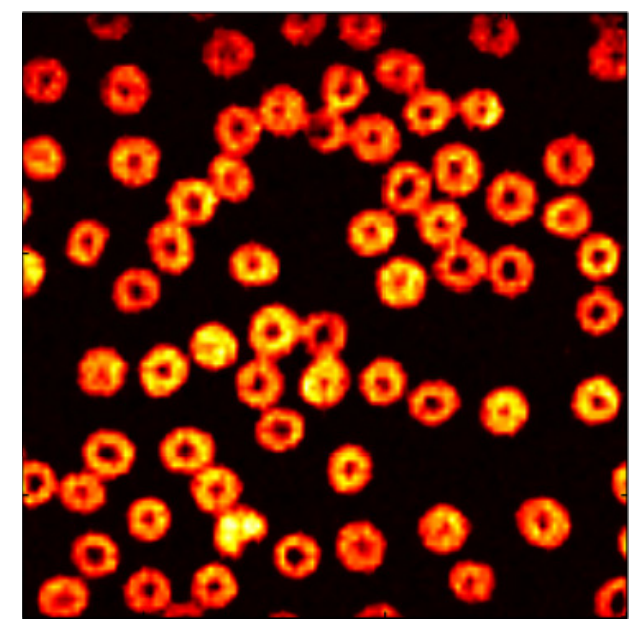

(b)

Fig. 9. (a) Simplified schematics of the CW LD-PAM setup, (b) photoacoustic image of red blood cells, (c) photoacoustic image on microspheres, (d) fluorescence image of (c), and (e) overlay of (c) and (d) (images adapted from Ref. 88). 


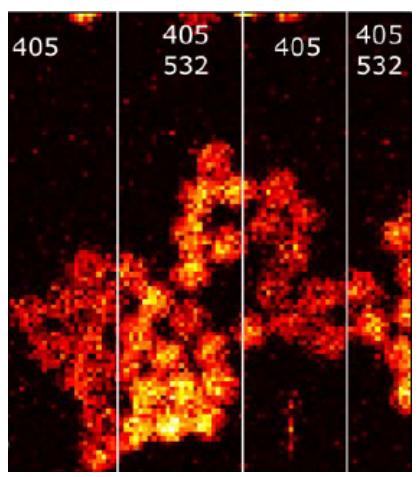

(c)

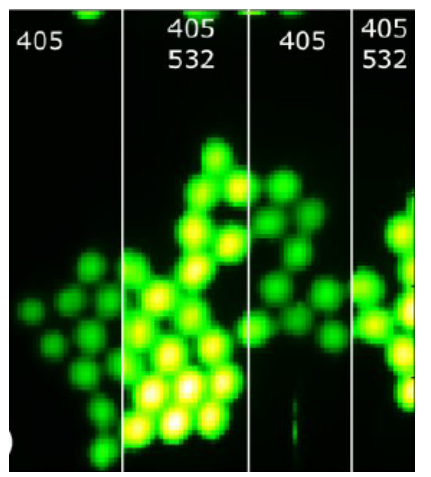

(d)

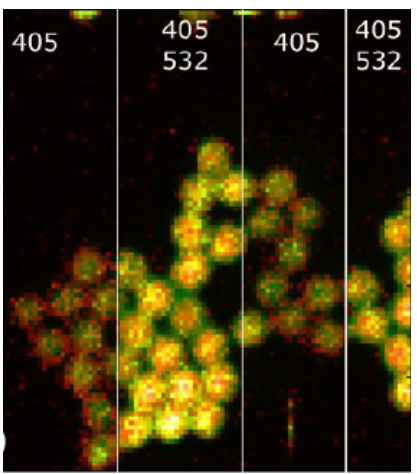

(e)

Fig. 9. (Continued)

M3 directs part of the light on a CCD camera which is exploited to get an overview image of the specimen. A needle hydrophone was employed to obtain the photoacoustic signals, and the luminescence light is led to an avalanche photodiode, respectively. The reconstructed image of erythrocytes was shown in Fig. 9(b), and the size of the image was $100 \times 100 \mu \mathrm{m}$ with a step size of $1 \mu \mathrm{m}$. Figures $9(\mathrm{c})$ and $9(\mathrm{~d})$ show the photoacoustic image and fluorescence image of fluorescent microspheres on a cover slip, and Fig. 9(e) was based on previous two images overlay. The signal intensity was strengthened after taking part in the $532 \mathrm{~nm}$ CW laser diode. Nevertheless, the photobleaching, optical absorption saturation, and temperature-dependent luminescence quantum yields of chromophores could result in a reduction in the corresponding signals.

\section{LED-Based PAI (LED-PAI)}

High-power LED as another potential alternative attributing to its low cost, miniature size, easy to integrate, besides, the efficient electronic driving and cooling system, and wide optical spectrum range from ultraviolet to NIR are peculiar strengths of LED. About a year ago, a high-power LED with a wavelength of $623 \mathrm{~nm}$ was employed in a LED-PAI system, as shown in Fig. 10(a). ${ }^{89}$ The LED with $0.01 \%$ duty cycle can provide a pulse duration of $200 \mathrm{~ns}$, repetition rate of $500 \mathrm{~Hz}$, and pulse energy of $9 \mu \mathrm{J}$. The sample was made up of three $1.4 \mathrm{~mm}$ diameter tubes and rotatable driven by a stepper motor. A $3.5 \mathrm{MHz}$ transducer was located on the opposite of the LED to capture the photoacoustic signal by averaging 5000 times. Figure 10(b) shows the reconstructed image of the tubes whose nominal values were within $0.1 \mathrm{~mm}$ of their nominal values. The penetration depth and image contrast ratio can reach up to $1.5 \mathrm{~cm}$ and 11.5 , respectively. The experimental results show that high-power LED could potentially be exploited in conjunction with NIR laser diode to obtain a wide range of biologically relevant wavelengths region for PAI.

In recent years, Hansen ${ }^{90}$ took initial steps toward LED-PAI. Although the series of studies have proved the feasibility of using high-power LED as

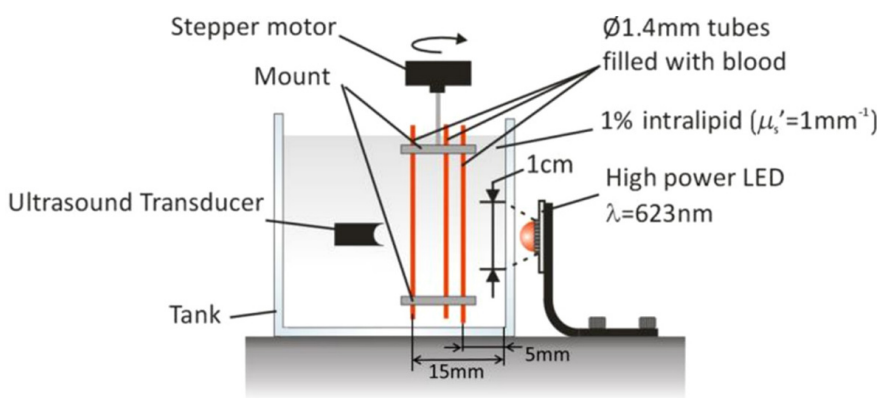

(a)

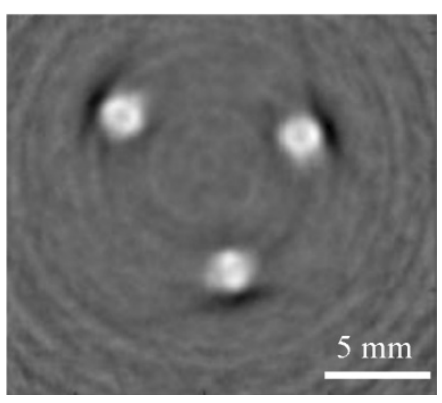

(b)

Fig. 10. (a) Schematic of pulsed LED-PAI system and (b) reconstructed photoacoustic image (images adapted from Ref. 89). 


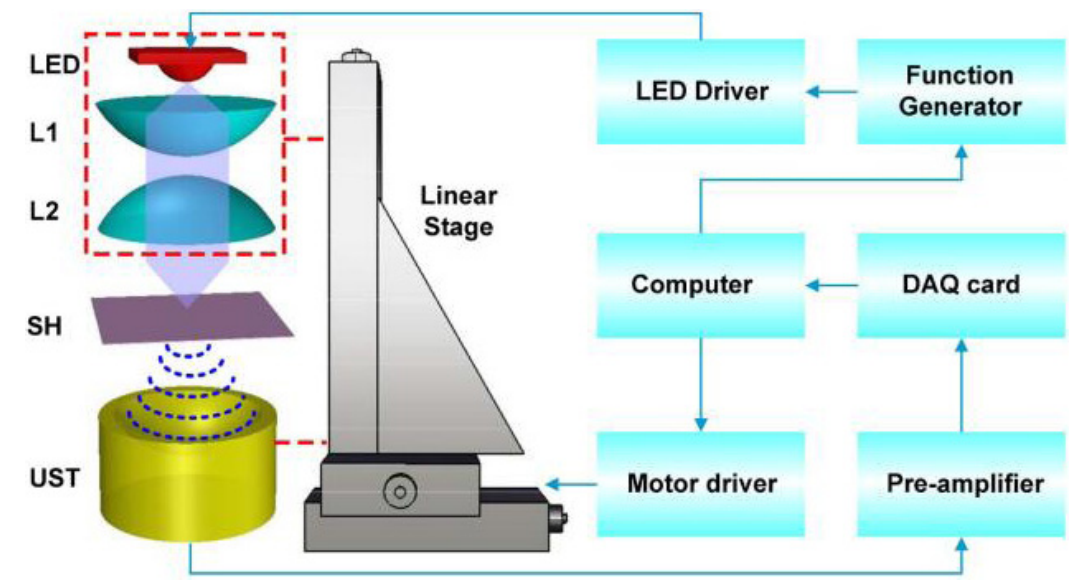

(a)

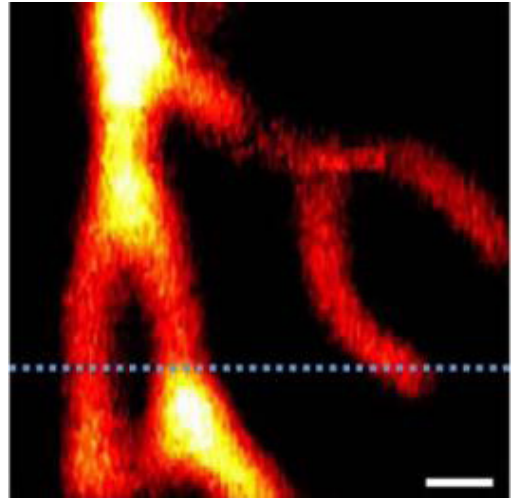

(b)

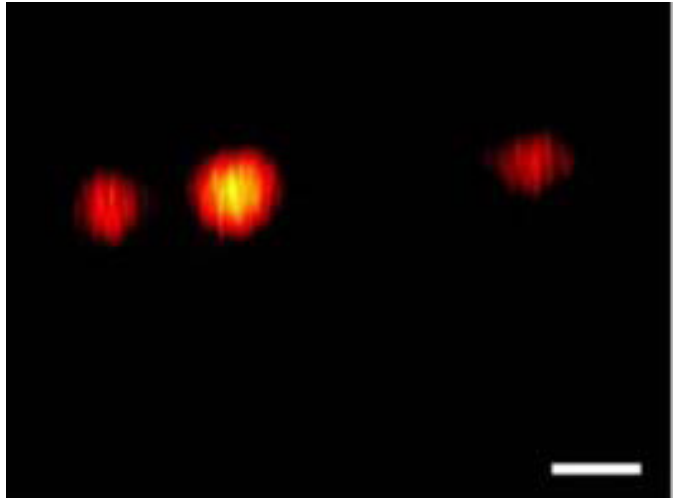

(c)

Fig. 11. (a) Schematic of pulsed LED-PAI system, (b) maximum amplitude projection image, and (c) photoacoustic images of the mouse ear the horizontal and depth directions (images adapted from Ref. 91).

an excitation source for PAI, they did not demonstrate the feasibility of in vivo tissue imaging with LED, and the low SNR of photoacoustic signals caused by the low light intensity reduced the possibility of in vivo imaging. Furthermore, real-time imaging capability of their system was limited by the LED's low repetition rate and the large averaging time. Recently, in vivo tissue imaging has been proved for the first time by LED-PAI. ${ }^{91}$ In this study, a super-fast switching high-current LED driving circuit with a rise-time of $2.2 \mathrm{~ns}$ and a falltime of $3.5 \mathrm{~ns}$ operated at $200 \mathrm{~ns}$ pulses, a repetition rate of $40 \mathrm{kHz}$ and provided $1.2 \mathrm{~W}$ output power at $405 \mathrm{~nm}$. The LED-PAI system is schematically shown in Fig. 11(a). Two aspheric lenses were used to focus the LED beams into zone, which was around $1 \mathrm{~mm}^{2}$. The SNR can be up to $14 \mathrm{~dB}$ when the photoacoustic signal was averaged 4000 times at a point in the vasculature of the mouse ear.
Figure 11(b) shows the maximum amplitude projection image and the cross-sectional image corresponding to the dotted line shown in Fig. 11(c). The in vivo images indicated the potential for portable biomedical imaging applications, and the handheld PAI devices would be possible with further exhibited by achieving a higher pulsed light energy LED array.

\section{Discussions}

Laser diode relative to Q-switch Nd:YAG is simple, compact, inexpensive, and highly power efficient, and the output peak power of a single element laser diode can be only up to several hundred watts. Composing a single element diode array, focussing the light, coding light excitation, or stacking diode bars is possible to image biological tissue with appropriate SNR and spatial resolution. For real-time 
imaging applications, it is quite easy for laser diode to operate at a high repetition frequency. Nevertheless, laser diode still has a number of drawbacks. First, most high-power laser diodes are not miniature enough to be packaged into an endoscope except when using a coupling fiber. Secondly, portable devices which are equipped with high-power laser diode are still deficient in the driving and cooling systems. Thirdly, commercially available high-power laser diodes which does not contain visible wavelength limit their applications in multispectroscopic or VIS PAI. In addition, focal spot is the crux of the emphasis in stacked laser diodes, and it will be the biggest hurdle of fiber coupling of light.

On the other hand, the limited availability of VIS laser diode is the advantage of LEDs. In the range of VIS wavelengths, the biology tissue which contains chromophores, like vasculum, will benefit from excitation source of LEDs. High-power LEDs are securable as another attractive alternative owing to relatively efficient electronic driving and cooling system and wide optical spectrum range from ultraviolet rays to NIR relative to laser diodes. However, the desired illumination of LED was usually an irregular shape with a large viewing angle, making it difficult to realize beam shaping. Furthermore, the application of optical fiber coupler in the LED-PAI system would be inefficient for portable devices. Additionally, nearly all LEDs were in wide spectrum, which limits the usability of LEDs in quantitative analysis, such as blood glucose detection.

In this review, we discussed the state of the art of PAI systems with the miniaturized laser diode and LED excitations, which has shown progress in many aspects: from preclinical to clinical, from bench to bedside, and from expensive to low cost. Generally, they still need to develop in image quality, penetration depth, and imaging speed. From the portability and reliability point of view, the practical and affordable PAI technology will profit from the replacement of bulky solid-state lasers by tiny semiconductor lasers.

\section{Acknowledgments}

This project is supported by the National Natural Scientific Foundation of China (11664011, 11304129), the Science and Technology Program of Jiangxi, China (20151BAB217025， 20132BBG70033， GJJ150790), and the Intramural Top-notch Youth Talent Program of JXSTNU, China (2013QNBJRC003).

\section{References}

1. A. G. Bell, "On the production and reproduction of sound by light," Am. J. Sci. 3(20), 305-324 (1880).

2. G. Lu, B. Fei, "Medical hyperspectral imaging: A review," J. Biomed. Opt. 19(1), 010901 (2014).

3. Y. Wu, Z. Tang, Y. Chi, L. Wu, "A simultaneous multi-probe detection label-free optical-resolution photoacoustic microscopy technique based on microcavity transducer," J. Biomed. Opt. 6(03), 1350027 (2013).

4. A. R. Kherlopian, T. Song, Q. Duan, M. A. Neimark, M. J. Po, J. K. Gohagan, A. F. Laine, "A review of imaging techniques for systems biology," BMC Syst. Biol. 2(1), 175-197 (2008).

5. M. Xu, L. V. Wang, "Time-domain reconstruction for thermoacoustic tomography in a spherical geometry," IEEE. Trans. Med. Imaging 21(7), 814$822(2002)$.

6. L. V. Wang, L. Gao, "Photoacoustic microscopy and computed tomography: From bench to bedside," Annu. Rev. Biomed. Eng. 16(16), 155-185 (2013).

7. L. V. Wang, S. Hu, "Photoacoustic tomography: In vivo imaging from organelles to organs," Science 335(6075), 1458-1462 (2012).

8. P. K. Upputuri, M. Pramanik, "Recent advances toward preclinical and clinical translation of photoacoustic tomography: A review," J. Biomed. Opt. 22(4), 041006 (2017).

9. X. Cai, Y. S. Zhang, Y. Xia, L. V. Wang, "Photoacoustic microscopy in tissue engineering," Mater. Today 16(3), 67-77 (2013).

10. P. Beard, "Biomedical photoacoustic imaging," Interf. Focus 1(4), 602 (2011).

11. S. Mallidi, G. P. Luke, S. Emelianov, "Photoacoustic imaging in cancer detection, diagnosis, and treatment guidance," Trends Biotechnol. 16(7), 213-221 (2011).

12. V. Ntziachristos, "Going deeper than microscopy: The optical imaging frontier in biology," Nat. Med. 7(8), 603-614 (2010).

13. C. Kim, C. Favazza, L. V. Wang, "In vivo photoacoustic tomography of chemicals: High-resolution functional and molecular optical imaging at new depths," Chem. Rev. 110(5), 2756-2782 (2010).

14. L. Leggio, D. C. Gallego, S. B. Gawali, M. Sánchez, S. Rodriguez, M. Osiński, J. Sacher, G. Carpintero, H. Lamela, "System analysis of wavelength beam combining of high-power diode lasers for photoacoustic endoscopy," Proc. SPIE 9888, 988807 (2016).

15. A. Hariri, A. Fatima, N. Mohammadian, N. Bely, M. Nasiriavanaki, "Towards low cost photoacoustic 
spectroscopy system for evaluation of skin health," Proc. SPIE 9976, 99760X (2016).

16. M. Erfanzadeh, Q. Zhu, "Low-cost laser scanning photoacoustic microscopy system with a pulsed laser diode excitation source," Proc. SPIE 10064, 10064R (2017).

17. P. K. Upputuri, S. K. Kalva, M. Moothanchery, M. Pramanik, "Pulsed laser diode photoacoustic tomography (PLD-PAT) system for fast in vivo imaging of small animal brain," Proc. SPIE 10064, $100645 \mathrm{O}(2017)$.

18. T. T. Wong, Y. Zhou, A. Garcia-Uribe, L. Li, K. Maslov, L. Li, L. V. Wang, "Use of a single xenon flash lamp for photoacoustic computed tomography of multiple-centimeter-thick biological tissue ex vivo and a whole mouse body in vivo," J. Biomed. Opt. 22(4), 041003 (2017).

19. G. Langer, A. Langer, B. Buchegger, J. Jacak, T. A. Klar, T. Berer, "Frequency domain optical resolution photoacoustic and fluorescence microscopy using a modulated laser diode," Proc. SPIE 10064, 1006426 (2017).

20. R. Li, P. Wang, L. Lan, P. L. Frank, C. J. Goergen, S. Chen, J. Cheng, "Assessing breast tumor margin by multispectral photoacoustic tomography," Biomed. Opt. Express 6(4), 1273-1281 (2015).

21. P. K. Upputuri, M. Pramanik, "Performance characterization of low-cost, high-speed, portable pulsed laser diode photoacoustic tomography (PLDPAT) system," Biomed. Opt. Express 6(10), 4118-4129 (2015).

22. X. Yang, A. Maurudis, J. Gamelin, A. Aguirre, Q. Zhu, L. V. Wang, "Photoacoustic tomography of small animal brain with a curved array transducer," J. Biomed. Opt. 14(5), 054007 (2009).

23. X. Yang, L. V. Wang, "Monkey brain cortex imaging by photoacoustic tomography," J. Biomed. Opt. 13(4), 044009 (2008).

24. M. Pramanik, G. Ku, C. Li, L. V. Wang, "Design and evaluation of a novel breast cancer detection system combining both thermoacoustic (TA) and photoacoustic (PA) tomography," Med. Phys. 35 (6), 2218-2223 (2008).

25. G. Ku, B. D. Fornage, X. Jin, M. Xu, K. K. Hunt, L. V. Wang, "Thermoacoustic and photoacoustic tomography of thick biological tissues toward breast imaging," Technol. Cancer Res. Treat. 4(5), 559565 (2005).

26. G. Ku, X. Wang, X. Xie, G. Stoica, L. V. Wang, "Imaging of tumor angiogenesis in rat brains in vivo by photoacoustic tomography," Appl. Opt. 44(5), 770-775 (2005).

27. X. Wang, Y. Pang, G. Ku, X. Xie, G. Stoica, L. V. Wang, "Noninvasive laser-induced photoacoustic tomography for structural and functional in vivo imaging of the brain," Nat. Biotechnol. 21(7), 803806 (2003).

28. R. A. Kruger, K. K. Kopecky, A. M. Aisen, D. R. Reinecke, G. A. Kruger, W. L. Kiser, "Thermoacoustic CT with radio waves: A medical imaging paradigm," Radiology 211(1), 275-278 (1999).

29. J. Y. Kim, L. Changho, P. Kyungjin, L. Geunbae, K. Chlhong, "Fast optical-resolution photoacoustic microscopy using a 2-axis water-proofing MEMS scanner," Sci. Rep. 5, 7932 (2014).

30. S. L. Chen, L. J. Guo, X. Wang, "All-optical photoacoustic microscopy," Photoacoustics 3(4), 143150 (2015).

31. B. Ning, M. J. Kennedy, A. J. Dixon, N. Sun, R. Cao, B. T. Soetikno, R. Chen, Q. Zhou, K. K. Shung, J. A. Hossack, S. Hu, "Simultaneous photoacoustic microscopy of microvascular anatomy, oxygen saturation, and blood flow," Opt. Lett. 40(6), 910-913 (2015).

32. L. Zeng, D. Xing, H. Gu, D. Yang, S. Yang, L. Xiang, "High antinoise photoacoustic tomography based on a modified filtered backprojection algorithm with combination wavelet," Med. Phys. 34 (2), 556-563 (2007).

33. B. Dong, H. Li, Z. Zhang, K. Zhang, S. Chen, C. Sun, H. Zhang, "Isometric multimodal photoacoustic microscopy based on optically transparent micro-ring ultrasonic detection," Optica 2(2), 169-176 (2015).

34. W. Song, W. Zheng, R. Liu, R. Lin, H. Huang, X. Gong, S. Yang, R. Zhang, L. Song, "Reflectionmode in vivo photoacoustic microscopy with subwavelength lateral resolution," Biomed. Opt. Express 5(12), 4235-4241 (2014).

35. T. Wang, S. Nandy, H. S. Salehi, P. D. Kumavor, Q. Zhu, "A low-cost photoacoustic microscopy system with a laser diode excitation," Biomed. Opt. Express 5(9), 3053-3058 (2014).

36. J. Xiao, Y. Li, W. Jin, K. Peng, Z. Zhu, B. Wang, "Photoacoustic endoscopy with hollow structured lens-focused polyvinylidine fluoride transducer," Appl. Opt. 55(9), 2301-2305 (2016).

37. C. Chen, Y. Zhao, S. Yang, D. Xing, "Mechanical characterization of intraluminal tissue with phaseresolved photoacoustic viscoelasticity endoscopy," Biomed. Opt. Express 6(12), 4975-4980 (2015).

38. J. M. Yang, C. Favazza, R. Chen, J. Yao, X. Cai, K. Maslove, Q. Zhou, K. K. Shung, L. V. Wang, "Simultaneous functional photoacoustic and ultrasonic endoscopy of internal organs in vivo," Nat. Med. 18(8), 1297-1302 (2012).

39. J. M. Yang, R. Chen, C. Favazza, J. Yao, C. Li, Z. $\mathrm{Hu}$, Q. Zhou, K. K. Shung, L. V. Wang, "A 2.5-mm diameter probe for photoacoustic and ultrasonic endoscopy," Opt. Express 20(21), 23944-23953 (2012). 
40. J. M. Yang, K. Maslov, R. Chen, H. C. Yang, Q. Zhou, K. Kirk Shung, L. V. Wang, "Volumetric photoacoustic endoscopy of internal organs: A phantom and in situ study," Proc. SPIE 7564, 75640D (2010).

41. J. M. Yang, K. Maslov, H. C. Yang, Q. Zhou, K. K. Shung, L. V. Wang, "Photoacoustic endoscopy," Opt. Lett. 34(10), 1591-1593 (2009).

42. Y. Fan, A. Mandelis, G. Spirou, I. A. Vitkin, "Development of a laser photothermoacoustic frequency-swept system for subsurface imaging: Theory and experiment," J. Acoust. Soc. Am. 116 (6), 3523-3533 (2005).

43. K. Maslov, L. V. Wang, "Photoacoustic imaging of biological tissue with intensity-modulated continuous-wave laser," J. Biomed. Opt. 13(2), 024006 (2008).

44. B. Lashkari, A. Mandelis, "Photoacoustic radar imaging signal-to-noise ratio, contrast, and resolution enhancement using nonlinear chirp modulation," Opt. Lett. 35(10), 1623-1625 (2010).

45. M. Li, P. Wang, "Optical resolution photoacoustic microscopy using a Blu-ray DVD pickup head," Proc. SPIE 8943, 894315 (2014).

46. E. M. Strohm, E. S. L. Berndl, M. C. Kolios, "High frequency label-free photoacoustic microscopy of single cells," Photoacoustics 1(3), 49-53 (2013).

47. S. Han, C. Lee, S. Kim, M. Jeon, J. Kim, C. Kim, "In vivo virtual intraoperative surgical photoacoustic microscopy," Appl. Phys. Lett. 103(20), 203702 (2013).

48. C. Zhang, K. Maslov, S. Hu, R. Chen, Q. Zhou, K. K. Shung, L. V. Wang, "Reflection-mode submicron-resolution in vivo photoacoustic microscopy," J. Biomed. Opt. 17(2), 020501 (2012).

49. G. Ku, K. Maslov, L. Li, L. V. Wang, "Photoacoustic microscopy with 2-microm transverse resolution," J. Biomed. Opt. 15(2), 021302 (2010).

50. S. Hu, K. Maslov, L. V. Wang, "Second-generation optical-resolution photoacoustic microscopy with improved sensitivity and speed," Opt. Lett. 36(7), 1134-1136 (2011).

51. W. Shi, S. Kerr, I. Utkin, J. Ranasinghesagara, L. Pan, Y. Godwal, R. J. Zemp, R. Fedosejevs, "Optical resolution photoacoustic microscopy using novel high-repetition-rate passively Q-switched microchip and fiber lasers," J. Biomed. Opt. 15(5), 056017 (2010).

52. L. Song, K. Maslov, R. Bitton, K. K. Shung, L. V. Wang, "Fast 3-D dark-field reflection-mode photoacoustic microscopy in vivo with a $30-\mathrm{MHz}$ ultrasound linear array," J. Biomed. Opt. 13(5), 054028 (2008).

53. H. F. Zhang, K. Maslov, G. Stoica, L. V. Wang, "Functional photoacoustic microscopy for high-resolution and noninvasive in vivo imaging," Nat. Biotechnol. 24(7), 848-851 (2006).
54. K. Maslov, G. Stoica, L. V. Wang, "In vivo darkfield reflection-mode photoacoustic microscopy," Opt. Lett. 30(6), 625-627 (2005).

55. S. A. Ermilov, T. Khamapirad, A. Conjusteau, M. H. Leonard, R. Lacewell, K. Mehta, T. Miller, A. A. Oraevsky, "Laser optoacoustic imaging system for detection of breast cancer," J. Biomed. Opt. 14(2), 024007 (2009).

56. Y. Zhao, S. Yang, "Photoacoustic viscoelasticity imaging of biological tissues with intensity-modulated continuous-wave laser," J. Innov. Opt. Health Sci. 6(04), 1350033 (2013).

57. X. L. Deán-Ben, D. Razansky, "Portable spherical array probe for volumetric real-time optoacoustic imaging at centimeter-scale depths," Opt. Express 21(23), 28062-28071 (2013).

58. H. Ke, T. N. Erpelding, L. Jankovic, C. Liu, L. V. Wang, "Performance characterization of an integrated ultrasound, photoacoustic, and thermoacoustic imaging system," J. Biomed. Opt. 17(5), 056010 (2012).

59. G. M. R. Kolkman, W. Steenbergen, T. G. van Leeuwen, "In vivo photoacoustic imaging of blood vessels with a pulsed laser diode," Lasers Med. Sci. 21(3), 134-139 (2006).

60. M. P. Mienkina, C. S. Friedrich, N. C. Gerhardt, M. F. Beckmann, M. F. Schiffner, M. R. Hofmann, G. Schmitz, "Multispectral photoacoustic coded excitation imaging using unipolar orthogonal Golay codes," Opt. Express 18(9), 9076-9087 (2010).

61. C. Willert, B. Stasicki, J. Klinner, S. Moessner, "Pulsed operation of high-power light emitting diodes for imaging flow velocimetry," Meas. Sci. Technol. 21(7), 129-247 (2010).

62. C. E. Willert, D. M. Mitchell, J. Soria, "An assessment of high-power light-emitting diodes for high frame rate schlieren imaging," Exp. Fluids 53(2), 413-421 (2012).

63. K. Daoudi, S. Tisserand, W. Steenbergen, "Handheld probe for portable high frame photoacoustic/ultrasound imaging system," Proc. SPIE 8581(3), 858121 (2013).

64. C. Zhang, K. Maslov, L. V. Wang, "Subwavelengthresolution label-free photoacoustic microscopy of optical absorption in vivo," Opt. Lett. 35(19), 31953197 (2010).

65. J. Yao, L. Song, L. V. Wang, "Photoacoustic microscopy: Superdepth, superresolution, and superb contrast," IEEE Pulse 6(3), 34-37 (2015).

66. X. Dai, H. Yang, H. Jiang, "Low-cost high-power light emitting diodes for photoacoustic imaging," Proc. SPIE 10064, 10064E (2017).

67. Y. Shigeta, T. Agano, N. Sato, H. Nakatsuka, K. Kitagawa, T. Hanaoka, K. Morisono, C. Tanaka, "Detection of ICG at low concentrations by 
photoacoustic imaging system using LED light source," Proc. SPIE 10064, 100644X (2017).

68. M. L. Li, P. H. Wang, "Optical resolution photoacoustic microscopy using a Blu-ray DVD pickup head," Proc. SPIE 8943, 894315 (2014).

69. L. V. Wang, S. Hu, "Photoacoustic tomography: In vivo imaging from organelles to organs," Science 335(6075), 1458-1462 (2012).

70. K. Maslov, H. F. Zhang, S. Hu, L. V. Wang, "Optical-resolution photoacoustic microscopy for in vivo imaging of single capillaries," Opt. Lett. 33(9), 929-931 (2008).

71. J. M. Yang, C. Favazza, R. Chen, J. Yao, X. Cai, K. Maslov, Q. Zhou, K. K. Shung, L. V. Wang, "Simultaneous functional photoacoustic and ultrasonic endoscopy of internal organs in vivo," Nat. Med. 18(8), 1297-1302 (2012).

72. D. Wang, Y. Wang, W. Wang, D. Luo, U. Chitupi, J. Geng, Y. Zhou, L. Wang, J. F. Lovell, J. Xia, "Deep tissue photoacoustic computed tomography with a fast and compact laser system," Biomed. Opt. Express 8(1), 112-123 (2017).

73. L. Zeng, G. Liu, Z. Ren, Z. Huang, D. Yang, "Design of a portable noninvasive photoacoustic glucose monitoring system integrated laser diode excitation with annular array detection," Proc. SPIE 7280, $72802 \mathrm{~F}(2008)$.

74. D. Yang, L. Zeng, C. Pan, X. Zhao, X. Ji, "Noninvasive photoacoustic detecting intraocular foreign bodies with an annular transducer array," Opt. Express 21(1), 984-991 (2013).

75. L. Zeng, G. Liu, Z. Ren, Z. Huang, B. L. Shao, "Design of high-resolution photoacoustic imaging system based on LabVIEW station," Acta Photon. Sin. 37(7), 1436-1440 (2008).

76. L. Zeng, G. Liu, D. Yang, J. Dong, "Compact optical-resolution photoacoustic microscopy system based on a pulsed laser diode," Chin. J. Laser 40, 131-136 (2014).

77. L. Zeng, G. Liu, D. Yang, X. Ji, "3D-visual laserdiode-based photoacoustic imaging," Opt. Express 20(2), 1237-1246 (2012).

78. T. J. Allen, P. C. Beard, "Pulsed near-infrared laser diode excitation system for biomedical photoacoustic imaging," Opt. Lett. 31(23), 3462-3464 (2006).

79. T. J. Allen, P. C. Beard, "Dual wavelength laser diode excitation source for 2D photoacoustic imaging," Proc. SPIE 6437, 64371U (2007).

80. P. K. Upputuri, M. Pramanik, "High-speed preclinical brain imaging using pulsed laser diode based photoacoustic tomography (PLD-PAT) system," Proc. SPIE 9708, 97084R (2016).

81. K. Daoudi, P. J. van den Berg, O. Rabot, A. Kohl, S. Tisserand, P. Brands, W. Steenbergen, "Handheld probe integrating laser diode and ultrasound transducer array for ultrasound/photoacoustic dual modality imaging," Opt. Express 22 (21), 26365-26374 (2014).

82. P. J. van den Berg, R. Bansal, K. Daoudi, W. Steenbergen, J. Prakash, "Preclinical detection of liver fibrosis using dual-modality photoacoustic/ultrasound system," Biomed. Opt. Express 7(12), 5081 (2016).

83. L. Zeng, G. Liu, D. Yang, X. Ji, "Portable opticalresolution photoacoustic microscopy with a pulsed laser diode excitation," Appl. Phys. Lett. 102(5), 053704 (2013).

84. L. Zeng, G. Liu, D. Yang, X. Ji, "Cost-efficient laserdiode-induced optical-resolution photoacoustic microscopy for two-dimensional/three-dimensional biomedical imaging," J. Biomed. Opt. 19(7), 076017 (2014).

85. T. Wang, S. Nandy, H. S. Salehi, P. D. Kumavor, Q. Zhu, "A low-cost photoacoustic microscopy system with a laser diode excitation," Biomed. Opt. Express 5(9), 3053-3058 (2014).

86. M. Erfanzadeh, H. S. Salehi, P. Kumavor, Q. Zhu, "Improvement and evaluation of a low-cost laser diode photoacoustic microscopy system for ovarian tissue imaging," Proc. SPIE 9708, 97083I (2016).

87. L. Zeng, Z. Piao, S. Huang, W. Jia, Z. Chen, "Labelfree optical-resolution photoacoustic microscopy of superficial microvasculature using a compact visible laser diode excitation," Opt. Express 23(24), 3102631033 (2015).

88. G. Langer, T. Berer, "Non-linear photoacoustic and fluorescence microscopy using a modulated laser diode," Proc. SPIE 9708, 97082K (2016).

89. T. J. Allen, P. C. Beard, "High power visible light emitting diodes as pulsed excitation sources for biomedical photoacoustics," Biomed. Opt. Express 7 (4), 1260-1270 (2016).

90. R. S. Hansen, "Using high-power light emitting diodes for photoacoustic imaging," Proc. SPIE 7968, 79680A (2011).

91. X. Dai, H. Yang, H. Jiang, "In vivo photoacoustic imaging of vasculature with a low-cost miniature light emitting diode excitation," Opt. Lett. 42(7), 1456-1459 (2017). 